COMMENT. The authors cite reports of anti-MuSK MG mainly in middle-aged female adults, and a frequency of anti-MuSK Ab in AChR seronegative MG in $38 \%$ to $47 \%$ of cases. The present late infantile case suggests a wider spectrum of disease. The response to immunotherapies was thought to rule out a diagnosis of congenital MG.

A study of 77 juvenile myasthenia gravis patients, evaluated over a period of 34 years at the National Institute of Mental Health, Bangalore, India, and compared to 290 patients with MG onset after 15 years of age, showed $30 \%$ had ocular myasthenia and the remainder had generalized MG at onset (Ashraf VV et al. Acta Neurol Scand Aug 2006;114:119-123). MG was familial in $10(13 \%)$, with siblings affected. Treatment with corticosteroids was used in $52(67 \%)$, and 5 received azathioprine in addition. Thymectomy was performed in 11 , thymic histology showing hyperplasia in 8 and thymoma in 1 . In 64 patients with follow-up information (mean 6.2 years; range 6 mos to $25 \mathrm{yrs),} 10$ (18.5\%) had complete remission for at least one year without therapy, $25(39 \%)$ were asymptomatic, $28(44 \%)$ were partially improved, and $9(14 \%)$ where unchanged or worse. Two died, and 4 had crises.

\title{
A NOVEL VARIANT OF AUTOSOMAL RECESSIVE LOWER MOTOR NEURON DISEASE
}

A genome-wide linkage analysis to map the underlying genetic defect was performed in a consanguineous African family with five patients affected with a novel variant of autosomal recessive lower motor neuron disease (LMND), in a study at Hopital Necker Enfants Malades and other centers, Paris, France. In 4 of the 5 patients having a severe phenotype, symptoms appeared during infancy ( 2 to 3.5 years), with proximal muscle weakness predominating in the lower limbs and early involvement of foot and hand muscles. Paralysis spread to become generalized, except for the cranial nerves, and by age 16 years, patients were tetraplegic with areflexia, contractures, and scoliosis, and required assisted respiratory ventilation. Intelligence was preserved. The mild phenotype in one patient had a delayed onset (11.5 years), a moderate generalized weakness, and a slower course. Genetic testing ruled out linkage to the 5q13 and 21q22 chromosomal regions and SMN1 and SODI loci. This novel LMND variant was assigned to a locus on chromosome 1p36. (Maystadt I, Zarhrate M, Leclair-Richard D et al. A gene for an autosomal recessive lower motor neuron disease with childhood onset maps to 1p36. Neurology July (1 of 2) 2006;67:120-124). (Reprints: Dr L Viollet, Hopital Necker Enfants Malades, 149 rue de Sevres, F-75743 Paris Cedex 15, France).

COMMENT. Studies are underway to identify the gene involved in this novel variant of autosomal recessive LMND. Numerous phenotypes of childhood-onset LMND have been described, the most common being the proximal spinal muscular atrophy (SMA), linked to the SMNI gene. Kugelberg-Welander disease (SMA type III) has the same range of age at onset (after age of walking) as this novel variety, but the foot and hand paralysis, severe respiratory involvement, and lack of linkage to chromosome $5 \mathrm{q} 13$ that characterize the variant exclude the SMA III diagnosis. 